\title{
L'EXPLOITATION MINIERE ET LA PROTECTION DE LA MAIN-D'CEUVRE LOCALE A KOLWEZI
}

\author{
Par le Chef de Travaux Esdras ILUNGA NGUZA et l'Assistant Joseph MULAND \\ KATAL.
}

\section{INTRODUCTION GENERALE}

La main-d'œuvre, travail de l'ouvrier dans la confection d'un ouvrage, concernant le contrat à durée déterminée par l'achèvement de cet ouvrage, ou l'ensemble des salariés d'une entreprise, d'un pays concernant le contrat à durée indéterminée.

En effet, compte tenu de l'internationalisation des entreprises minières, marquée par la présence des employés expatriés dans les sociétés congolaises. Cette réalité pose souvent de grands problèmes relatifs à la protection de la main-d'œuvre nationale. Nul n'ignore que la ville de Kolwezi est un carrefour pour les investissements, un terrain de référence d'affaire pour les opérateurs économiques dans le domaine minier, à cause de ses ressources naturelles telles que : de l'or, le cobalt, le cuivre, le fer, la malachite, le zinc, etc. Ces ressources intéressent plus d'une personne : nationaux, expatriés et même les multinationaux bailleurs de fonds.

Ainsi, le secteur minier qui jadis était le monopole de l'Etat, depuis la promulgation du code minier, les particuliers tant nationaux qu'étrangers ayant un permis d'exploitation; ont créé des entreprises minières dont les étrangers bailleurs de fonds, dans la plus part de cas, ont une forte participation dans la constitution du capital de l'entreprise. A Kolwezi un mouvement de capitaux financés par les étrangers a été à la base de la création de plus ou moins 150 entreprises minières au cours de cette dernière décennie. Dans ces entreprises on y trouve une forte compétition de la main d'œuvre tant nationale qu'étrangère.

D'où l'impact de notre étude qui marque son importance sur l'exploitation minière et la protection de la main-d'œuvre locale. Peu importe sa nature, nationale ou immigrée, la main d'œuvre nécessite une protection sanctionné de la loi, contre des abus du patronat. Considérant l'expansion des entreprises minières à Kolwezi, la curiosité scientifique nous a poussés de mener une investigation, pour nous rendre compte des mesures de protection de la main-d'œuvre nationale en générale et locale en particulier.

En effet, nous avons été motivés, dans le choix de cette thématique par cette politique du plein emploi et de la priorité d'embauche réservée aux congolais. Cette politique consolide la protection de la main-d'œuvre nationale en vue de résorber tant soit peu le chaumage. Mais nous avons remarqué à Kolwezi, malgré l'expansion des entreprises minières, une forte crise de chômage plane dans la vie sociale de plus de soixante pourcent des autochtones de Kolwezi. La conséquence de cette crise de chaumage récurrente, se caractérise par la pauvreté. Ceci nous a poussés à étudier les problèmes liés à la protection de la maind'œuvre nationale. 
La R.D.C. a ratifié certaines conventions relatives à la non discrimination d'embauche de telle sorte que nul ne peut conditionner l'engagement du demandeur d'emploi à certain nombre d'éléments discriminatoires tels que : la nationalité, l'origine, le sexe, la race et ceux en vertu des droits fondamentaux qui se fondent sur la notion d'égalité de tous.

Cependant, tout Etat souverain ne s'exprime valablement que par sa propre législation nationale, question de promouvoir et de protéger surtout l'intérêt communautaire. Sans porter préjudice au principe de la non discrimination, l'article 6 du code du travail en son alinéa1 dispose que : «la capacité d'une personne d'engager ses services est régie par la loi nationale». Ainsi, les conventions fixent et organisent les modalités d'embauche.

Dans la pratique, l'on remarque un bon nombre des travailleurs étrangers et allochtones, alors que presque $80 \%$ des autochtones de Kolwezi, n'ont pas d'emploi. C'est à dire que la main-d'œuvre locale n'est pas parfaitement protégée contre la concurrence extérieure. Dès lorsque dans la plus part des sociétés minières privées, nous observons de fois un taux élevé de plus de $15 \%$ des expatriés, soit certains étrangers exercent les taches interdites par la loi, alors que réservés uniquement aux seuls patriotes congolais. Cette considération doit interpeller les autorités à veiller sur l'effectivité des lois relatives à la protection de la main d'œuvre nationale.

De tout ce qui précède, le centre de notre préoccupation est celui de savoir comment les sociétés minières qui connaissent du jour au jour, une inflation considérable dans la ville minière de Kolwezi; respectent les conditions prévues en matière d'embauche. Ainsi, par une analyse statistique du nombre d'employé nationaux et étranger sans préjudice des spécialités; on saura rapidement le degré de protection de la main-d'œuvre tant locale qu'étrangère.

A l'esquisse de notre travail, l'esprit scientifique nous a conduits, à la recherche et à la consultation de différents documents et ouvrages; pour situer de façon précise le sujet à traiter dans l'arsenal de la littérature existante dans cette thématique, à en dégager l'originalité. Certes, certains auteurs comme Paul Palster Man, nous montre dans son ouvrage que : « en Belgique, tout étranger qui préfère prester entant salarié, est tenu en principe d'avoir un permis de travail, il s'en suit en disant que bon nombre travailleurs étrangers sont cependant disposés de cette obligation ${ }^{1} »$.

En République Démocratique du Congo, l'ordre public intervient pour assurer la protection de la main-d'œuvre. Ainsi l'accès des étrangers au marché du travail est limité par la loi, il est prohibé à tout employeur d'avoir dans l'effectif de ses employés plus de $15 \%$ des personnes de la nationalité étrangère. Il existe une commission nationale de l'emploi des étrangers chargée de conseiller le ministre de travail sur la main-d'œuvre nationale contre la concurrence étrangère. Pour une précision à ce sujet, l'arrêté ministériel $\mathrm{N}^{\circ} 121$ COB/PTS/112/2005 du 26/ octobre 2005 fixant le maximum de pourcentage autorisé des employés étrangers au sein d'une firme dispose que : sans préjudice de l'article 185 alinéa 8 du code du travail, le pourcentage maxima du travailleurs étrangers pouvant agrée un em-

1 Paul Palster Man, et alii, Guide de la législation sociale, éd, syndicaliste, 2003, p213. 
ploi rémunéré en vertu d'un contrat de travail dans une firme par rapport à l'effectif total des travailleurs ne peuvent pas dépasser 5\% le maximum.

Ainsi, l'intervention du pouvoir public seraient capitales : pour prioriser l'emploi aux congolais; lutter contre la concurrence étrangère au profit de la main d'œuvre locale; pour monopoliser les travailleurs nationaux au sein des entreprises minières; la croissance de l'emploi pour les congolais et lutter contre le chômage des patriotes. Le seul souhait est que des investisseurs étrangers ou bailleurs des fonds, se lancent dans ce domaine miniers non pas pour avoir un nombre élevé de travailleurs étrangers, quoiqu'ils visent un intérêt spécialement déterminé, ils doivent comprendre et veiller au respect des lois nationales en matière d'embauche.

Pour arriver à cette analyse, nous avons utilisé la méthode exégétique qui nous a permis de démontrer, de saisir et de comprendre l'explication de la matière par et à travers les textes juridiques réglementant la protection de la main-d'œuvre nationale et l'étendu des entreprises minières en R.D.C. en générale et en particulier dans la ville de Kolwezi. La méthode dialectique nous a permis d'utiliser comme approche, sa loi de la contradiction qui nous a facilité de constater l'inadéquation du pourcentage prévu en matière d'embauche des nationaux et étrangers, pour voir le degré de protection.

Aussi l'interview libre nous a permis de recueillir divers renseignements, grâce aux questions que nous avons posées aux personnes qui exploitent ou qui sont concernée dans le secteur minier. Il s'agit notamment des employeurs, travailleurs, syndicats et toute autre personne possesseur d'information ou éléments en rapport avec cette thématique.

Cette étude se limite à la période allant de 2005 à ce jour. Cette période correspond à l'expansion des entreprises à Kolwezi, et la réglementation par le ministre ayant le travail dans ses attributions, du pourcentage maximal ne dépassant pas 5\%, de travailleurs étrangers dans les entreprises et sociétés en R.D.C.

Hormis l'introduction générale qui constitue le premier point et la conclusion générale ce travail s'est articulé autour de trois points. Le premier a été axé sur la définition des concepts, le deuxième a parlé de l'analyse fonctionnelle de mining à Kolwezi. Il comprend deux sous points dont l'un s'intéresse aux causes d'expansions de mining à Kolwezi et l'autre concerne l'impact de cette expansion. En fin le troisième a traité sur les enquêtes de la protection des mains-d'œuvre, menées dans quelques entreprises minières à Kolwezi.

\section{DEFINITION DES CONCEPTS}

Avant d'analyser le contenu du travail, nous avons relevé quelques concepts clés que couvre notre sujet que nous avons défini, pour garantir une bonne compréhension des faits juridiques à cerner. 


\section{II.1. La main-d'œuvre}

De manière générale la main-d'œuvre est « l'ensemble des salariés d'une entreprise, d'une région ou d'un pays $»^{2}$. La main-d'œuvre « est également définie comme la phrase incompréhensible des ouvriers dans la confection d'un ouvrage, ensemble des ouvriers nécessaires pour l'exécution d'un travail donné $»^{3}$.

\section{II.2. Typologie de la main-d'œuvre}

Il existe deux types de main-d'œuvre : la main-d'œuvre nationale et la main-d'œuvre étrangère (ou immigrée)

\section{II.2.1. La main-d'œuvre nationale}

Toutes les lois autour de ceci, ont pour finalité la restriction de droit des étrangers à accéder à l'emploi en République Démocratique du Congo, ces lois consacrent le principe de la priorité d'embaucher des congolais par rapport aux étrangers.

\section{II.2.2. La main-d'œuvre étrangère}

C'est un ensemble des expatriés ou étrangers, travailleurs au sein des entreprises du pays d'accueil. Ainsi en cas de concurrence entre un expatrié et un congolais, la préférence doit être portée au congolais; sauf si les travaux ne nécessitent pas une formation particulière.

\section{II.3. La protection}

C'est l'action de protéger, ou de mettre à l'abri d'un danger, d'un dommage.Ainsi, la protection de la main-d'œuvre signifie mettre à l'abri, mettre hors dangers la main-d'œuvre nationale contre la concurrence étrangère.

a) Protection de la main-d'œuvre nationale

Il est de principe que tout chef d'établissement ou entreprise publique ou privé est soumis à la formalité de déclaration d'ouverture ou fermeture d'établissement, il doit fournir à la division provinciale du travail de la prévoyance sociale et à l'inspection du travail au plus tard le 31 janvier de chaque année une déclaration de la situation de la main-d'œuvre qu'il emploi à la date du 1 janvier ${ }^{4}$.

2 Guillien et Vincent, J., Lexique des termes juridiques, 12éd Dalloz, 1999, paris.

3 Dictionnaire petit Larousse, 114, paris, p541.

4 Lettre 12/D.T.P.S/ CAB/0730/83 protection de la main d'œuvre nationale du 13 août 1983. 
Il ressort de l'analyse de la situation politique actuelle, que la plupart des Etats du monde entier, dont les ressortissant séjournent et travaillent en R.D.C. sont devenus souverains et entretiennent de rapport d'amitié avec notre Etat, en dehors de tout accord en matière d'emploi, une double attitude devra dès lors s'observer en cas d'embauche et de maintien en service des travailleurs étrangers selon que ces derniers sont cadres de maitrise ou qu'ils sont travailleurs ordinaires, relevant de la classification générale des emplois, en vigueur.

Quelques techniques ont été mises progressivement au point, pour la protection de la main-d'œuvre et aussi en vue de promouvoir l'emploi : stage des jeunes dans les entreprises et dans les Administrations publiques; cadre spécial temporaire, création d'un troisième circuit de travail, etc. La sécurité sociale en somme à elle-même a été utilisée comme un instrument d'emploi. L'abaissement de l'âge de retraite n'a d'autre but que d'inciter les travailleurs âgés à cesser prématurément leurs activités professionnelles pour libérer des emplois ${ }^{5}$.

\section{II.4. Le mining}

Il s'agit d'un mot anglais, qui signifie « exploitation minière ou pose de mine ou encore minage ». Alors que le décret du 20 mars 1893 ne s'est pas non plus attelé à définir les mines, mais plutôt à étendre plus ou moins la liste des catégories de ceux qu'on pouvait appeler "mines". C'est ainsi que son article premier considérait comme mines, toutes les substances minérales utilisables pour leur teneur en métaux, les substances utilisables pour leur teneur en soufre, les substances fossiles combustible ou bitumeuses, le sel gemme,les sels métalliques et également le diamant et les pierres précieuses. Le code des mines du Mali, en son article 116 considère les mines comme étant : « les gites des substances minérales comprenant toute ouverture en extraction fait dans le but de découvrir ou d'obtenir une substance minérale et les voies, travaux, machines, usines, bâtiments ou fourreaux sous ou sur la surface de terrain faisant parti d'une exploitation minière $»^{6}$.

Suivant l'usage auquel elles sont destinées, notamment pour les travaux d'utilités publics, certaines substances minières sont susceptibles d'être considérées comme des matériaux divers dans les limites fixées par l'autorisation administrative. CAZIN, estime qu'au sens usuel, on désigne sous le nom de « mines ", tout aussi bien les lieux d'où l'on extrait des métaux, des minéraux, que l'ensemble des travaux qui servent à l'exploitation d'un gîte de matières minérales $»^{7}$.

Il faut cependant, reconnaitre que le mérite d'une définition légale du terme « mines », sans procéder par l'élimination ni l'énumération, revient au législateur du 11 juillet 2002 qui a essayé, pour la toute première fois dans l'histoire du droit minier congolais, de fournir

5 MUKENDI WA FWANA, E, « Droit minier congolais », in jur. Congo, Kinshasa, p28.

6 MUKENDI wa FWANA, E., op.cit, p28.

7 CAZIN, M, les mines presses universitaires de France, collection « qui sais-je? », paris, 1951, p 09. 
le meilleur de lui-même en se hissant au rang des législateurs modernes et en donnant ainsi une définition à l'article premier comme étant : « tout gisement ou gisement artificiel des substances minérales classés en mines exploitables à ciel ouvert ou en souterrain, et/ou toute usine de traitement ou la transformation des produits de cette exploitation se trouvant dans le périmètre minier, y compris les installation et les matériels mobiliers et immobiliers affectés à l'exploitation ${ }^{8}$ ». Cette définition met en exergue des substances minérales qui peuvent se trouver classer en mines et selon qu'elles sont exploitées à ciel ouvert ou en souterrain.

a) Conditions préalables pour la création de mining

Les terrains de la surface sont occupés en vertu des titres désignés par le code minier. En effet pour occuper les terrains en question, il faut être minus des droits miniers. L'article 23 du code minier congolais qui traite de l'éligibilité aux droits miniers et de carrières énonce que : « sans préjudice des dispositions de l'article 27 du même code, sont éligibles aux droits miniers et des carrières :

$1^{\circ}$ Toute personne physique majeure de nationalité congolaise, ainsi que toute personne morale de Droit congolais, qui a son siège social et administratif dans le territoire national et dont l'objet social porte sur les activités minières.

$2^{\circ}$ Toute personne physique majeure de nationalité étrangère ainsi que toute personne morale de droit étranger.

$2^{\circ}$ Tout organisme à vocation scientifique $»$.

Par droit minier, il faut entendre toute prérogative d'effectuer la recherche et /ou l'exploitation des substances minérales classées en même conformément aux dispositions du présent code minier. Le permis de recherche, le permis d'exploitation, les permis d'exploitation de petite mine sont des droits miniers. Nous relevons cependant que, tous les droits miniers ne donnent pas la prérogative à leurs titulaires d'occuper les terrains de la surface, c'est par exemple le cas du permis de recherche qui ne confère à son titulaire aucun droit d'occuper, seul le permis d'exploitation qui donne lieu à l'occupation du terrain à exploiter.

En effet, il est également exigé que pour avoir un permis d'exploitation, il faut être une société commerciale créée conformément à la législation congolaise. Le permis de recherche en son bon sens, est octroyé si le périmètre demandé est disponible, si le requérant est la première personne à demander un droit minier sur le périmètre, le requérant est éligible aux droits miniers conformément aux dispositions de l'article 23 du code minier et ne tombe pas dans l'une des catégories des personnes non éligibles visées par le code minier.

Le titulaire du permis est tenu de se présenter auprès du Gouverneur de province et lui remettre les documents, il fera en plus la déclaration d'ouverture du centre de recherche ou d'exploitation auprès de la division provinciale des mines avec copie à la direction des mines. Il produira en suite les pièces suivantes : la copie du titre minier; la copie de l'autori-

8 La loi $\mathrm{N}^{\circ} 007 / 2002$ du 11 juillet 2002 portant code minier congolais. 
sation environnementale correspondant; l'identification des carrés à l'intérieure duquel le centre sera installé; le plan d'accès aux sites où sont installés les centres de recherche ou d'exploitation.

Le titulaire procède à se soumettre à l'approbation de la direction provinciale des mines avec copie à la division provinciale des mines, notamment le plan topographique du fond où les travaux seraient entreprendre au sein de ces centres ${ }^{9}$. Cependant, il est accorder à tout titulaire d'un droit minier un délai, endéans duquel, il doit commencer se travaux ou activités de recherche, de développement et de construction de la mine.

b) Caractère de mining dans la croissance économique

Nous pouvons relever cette croissance économique de mining à deux niveaux suivants : la visibilité de mining à leur impact socio-politico-économique et environnemental d'une part; et l'utilisation de la main- d'œuvre par les sociétés minières à Kolwezi d'autre part.

\section{DU FONCTIONNEMENT DES MINES A KOLWEZI}

III.1. Les causes d'expansion des mines à Kolwezi

III.1.1. Les causes juridiques

a) Aperçu historique

La République Démocratique du Congo a connu des nombreuses législations, dans le but de sauvegarder l'équilibre social. Ces législations ont été faites à ce propos en vue de permettre aux particuliers d'exercer leurs activités dans les formes légales. Sachant que la ville de Kolwezi est riche en gisement cuprifère et cobaltite. L'on a vue la nécessité d'une législation en cette matière et ce fut le Décret du 16 décembre 1910 modifié et complété par celui du 16 Avril 1919 apparus, juste pour réglementer la recherche et l'exploitation minière du sol du Katanga.

Ces décrets consistaient à harmoniser l'exploitation minière et d'éviter tout trouble quant à la jouissance du droit minier. Compte tenu de la représentation de la R.D.C. dans ses richesses du sol et du sous-sol et surtout pour mieux organiser une bonne gestion de la chose publique; il ya eu la nécessité de promulguer le décret du 24 septembre 1937 qui demeure en vigueur jusqu'en 1967 l'année à laquelle fut promulguée la loi relative aux mines et hydrocarbures ${ }^{10}$.

9 Décret $\mathrm{N}^{\circ} 038 / 2003 \mathrm{du}$ 26/03/2003 portant règlement minier, J.O.R.D.C. p176.

10 Ordo-loi N $\mathrm{N}^{\circ}$ 67/231 du $31 / 05 / 1967$ portant législation générale sur les mines et hydrocarbures. 
b) De la législation en vigueur

L'activité minière étant un point focal contribuant au développement du pays, a été l'objet d'une réalité ayant conduit à la modification de la première législation susmentionnée, qui présentait certaines imperfections qui n'étaient pas d'ordre public. Elle a favorisé l'existence de la deuxième législation de 1981 relative aux mines et hydrocarbures qui n'a pas résulté des innovations du fait qu'elle n'a pas permis la satisfaction d'intérêt général ${ }^{11}$.

Mais depuis 2002, le nouveau code minier a prévu les droits que les particuliers doivent posséder sur les concessions minières. Il est venu pallier les lacunes que contenaient les autres législations antérieures dans le secteur minier en l'occurrence : l'imprévision des droits de particuliers sur les mines et les avantages des investissements en vertu desquels, les investisseurs lancent leurs capitaux.

Ce nouveau code minier ouvre à tout celui qui désire œuvrer dans le secteur minier, les portes d'y accéder à condition de respecter les dispositions qui y sont prévues. La législation en matière d'investissement ne vise pas seulement la règlementation de l'économie mais également la promotion de celle-ci.

En effet, le code des investissements n'est pas une loi intimidatrice, c'est plutôt celle qui se manifeste par les opérations de la protection des investisseurs. La R.D.C. s'engage à assurer un traitement juste et équitable, conformément aux principes du droit international, aux investisseurs et aux investissements effectués sur son territoire. Et à faire en sorte que l'exercice du droit ainsi reconnu, ne soit entravé ni en droit ni en fait. L'article 34 alinéa1 de la constitution congolaise du 18/02/2006, garantit les droits de propriété individuelle ou collective acquis par un investisseur.

La loi sur l'investissement interdite qu'un investissement soit directement ou indirectement, dans sa totalité ou en partie, nationalisé ou exproprié par une loi nouvelle et ou une décision d'une autorité locale ayant le même effet, excepté pour des motifs d'utilité publique et moyennant le payement d'une juste et équitable indemnité compensatoire.

Le code des investissements accorde aux investisseurs agréés les avantages douaniers, fiscaux et parafiscaux y afférents pour une durée de trois ans lorsqu'ils sont dans la région économique de la ville province de Kinshasa, quatre ans lorsqu'ils exercent leurs activités dans la région économique du Bas- Congo, Lubumbashi, Likasi et Kolwezi et cinq ans lorsqu'ils sont dans la région économique d'autres provinces ${ }^{12}$.

Sans préjudice de la redevance administrative, les investissements d'utilité publique bénéficient de l'exonération totale des droits de toute importation pour les machines, l'outillage, les matériels neufs, les pièces de rechanges de premières dotations ne dépassant pas $10 \%$ de la valeur du coût d'achat du dits équipements. Dès lors que leurs demandes sont

11 Ordo-loi $\mathrm{N}^{\circ} 81 / 013$ de la 02/04/1982, portant législation générale sur les mines et hydrocarbures.

12 Article 3 de la loi $\mathrm{N}^{\circ} 004 / 2002$ du 21/02/2002 portant code des investissements congolais. 
approuvées par l'agence nationale pour la promotion des investissements (A.NA.P.I. en sigle $)^{13}$.

Les personnes physiques ou morales étrangères reçoivent un traitement identique à celui des personnes physiques ou morales congolaises ${ }^{14}$. Sous réserve de l'application du même principe d'égalité de traitement par l'Etat dont la personne physique ou morale et même étrangère concernée est ressortissante; les personnes physiques ou morales reçoivent également les mêmes traitements.

Sous réserve des dispositions du traité et accord conclus par la R.D.C., ces traitements ne s'étendent toutefois pas aux privilèges que la R.D.C. Accorde aux nationaux ou société d'Etat tiers en vertu de sa participation à une zone de libre échange, une union douanière, un marché commun ou toute autre forme d'organisation économique, régionale,... excepté pour la fiscalité. La R.D.C. s'engage à assurer un bon traitement juste et adéquat en corrélation aux principes du droit international.

\section{III.1.2. Les causes politiques}

a) Aperçu historique

Depuis son accession à l'indépendance, le 30 juin 1960, la République Démocratique du Congo est confortée à des crises politiques récurrentes dont l'issu des causes fondamentales est la contestation de la légitimité des institutions et de leurs animateurs. Cette contestation a pris un relief particulier avec les guerres qui ont déchirées le pays de 1996 à 2003.

\section{b) Mécanismes}

L'on a constaté une chaine des législations sur les mines, malheureusement ces législations antérieures se trouvaient à mi-chemin. Chose incroyable en 2002 l'apparition d'un nouveau code minier a pu réaffirmer la souveraineté de l'Etat sur les richesses minières, la libéralisation de l'exploitation minière artisanale de certaines ressources minérales et les investissements dans le programme agricole ou social.

Dans ces textes, l'on s'est rendu compte de la participation obligatoire et sans contrepartie financière de l'Etat dans les industries minières. Cependant, après avoir recherché le fondement de cette participation, depuis l'époque coloniale jusqu'aujourd'hui, à travers les dispositions légales et conventionnelles, il n'a été relevé aucun fondement de l'engagement de l'Etat dans le secteur minier. Et donc, le nouveau code minier a donné une définition claire du rôle de l'Etat. En effet, les exigences des parties étaient de voir transformer le rôle de l'Etat c'est-à-dire de la production en régulateur.

13 Article 3 alinéa 2 de la même loi.

14 Code larcier, droit commercial et économique, T3 Vol2 Afrique, éd Bruxelles 2003, p568. 
c) De la responsabilité de l'Etat

Le nouveau code minier de 2002 pose le principe de voir l'Etat se désengager dans le secteur minier, il se voit quasiment interdit d'influencer les libres cours à l'initiative privée. L'Etat assure la mise en valeur des substances minérales dont il est propriétaire en faisant appel à l'initiative privée conformément aux dispositions du présent code minier.

L'on observe que l'Etat doit rester imbriquer principalement dans une case d'éclaireur et de surveillant en matière d'exploitation minière, c'est-à-dire, assurer un bon encadrement juridique et administratif nécessaire pour permettre aux particuliers d'exercer en toute foi et liberté. La même disposition ouvre une voie à l'Etat, soit de se livrer à des activités d'investigation du sol ou du sous-sol ${ }^{15}$.

S'agissant du premier cas, l'on notera que les substances minérales sont épuisables qui nécessitent l'intervention du pouvoir public pour maitriser la cartographie géologique et promouvoir les recherches géologiques afin de mettre à la disposition des particuliers des informations sur l'existence éventuelle de gisements miniers sur le territoire national, moyennant paiement des taxes ou redevances fiscales.

L'Etat peut se livrer à travers des organismes spécialisés crées à cet effet, des activités d'investigations du sol ou du sous-sol dans le seul but d'améliorer la connaissance géologique du territoire national ou à des objectifs scientifiques qui ne requièrent pas l'obtention d'un droit minier ou des carrières ${ }^{16}$.

\section{III.1.3. Les causes économiques}

a) Historique

Au cours des années 1967-1974, l'économie de la République Démocratique du Congo, était tournée fortement vers l'extérieur, mais elle satisfaisait tant bien que mal, les besoins et les intérêts de la population. Cette période était caractérisée par une croissance liée aux importants investissements d'entreprises multinationales dans le secteur minier.

Une série d'événements en cette période est apparue en l'occurrence la Zaïrianisation, la fermeture des voies de sortie des produits miniers vers les pays d'Afrique Australe, la flambée des prix du pétrole, l'effondrement de la mine de KAMOTO, la baisse du prix du cuivre; vont entrainer la faillite du secteur minier de la vie économique du Pays. La dégradation de la vie socio-économique dans certaines villes du pays et plus fortement celles du Katanga avait pour conséquence, la détérioration du niveau de vie des habitants.

15 ILUNGA Watuil, C, De l'Etat et des exploitants miniers face au droit de l'environnement en RDC, thèse de doctorat, UNILU, 2013, inédit, p329.

16 Loi $\mathrm{N}^{\circ} 007 / 2002$ du 11/07/2002 portant code minier, in J.O.Nº spécial, 15/07/2002, p38. 


\section{b) Observation}

La diminution considérable du nombre de salariés, est allée de paire avec l'expansion des activités économiques informelles, pour multiplier les sources du revenu. C'est pourquoi, une étude de l'observation urbaine du changement montre qu'avec l'avènement du nouveau code minier et celui des investissements, une multiplication rapide des sociétés minières tant privées que publiques.

Les programmes mis en œuvre par les institutions financières internationales dans le secteur, se caractérisent par l'implantation de reformes macroéconomiques et structurelles destinées à favoriser l'entrée de capitaux étrangers pour impulser une relance économique. Ces politiques économiques de libéralisation basées sur la croissance seraient susceptibles de promouvoir le développement des pays et aussi leur donner les moyens de consolider le processus démocratique et de lutter contre la pauvreté.

Pour y arriver, l'objectif reste ce lui de convertir la République Démocratique du Congo en une destination attrayante pour les investisseurs. Mais malgré tout, il est seulement un centre de la stratégie des bailleurs de fonds et gouvernement qui misent principalement sur les ressources naturelles du pays (mines, foresterie, et agriculture).

\section{c) De l'intervention}

La guerre a mis les projets de développement économique du pays en cascade, mais dès 2001, dans un laps de temps, on a constaté un retour en force des organisations financières internationales au Congo ${ }^{17}$. L'implication des institutions de Brettons Wood en République Démocratique du Congo, est présentée par les investisseurs privés comme une avancée significative pour le pays en termes de garantie de stabilité et de sécurité financière. La chronologie des événements qui va suivre, nous permet d'illustrer par quelles étapes les reformes amorcées dans tous les secteurs de l'économie du pays, se mettent en place dans le secteur minier.

\section{d) Tournant décisif}

Depuis 1999, la République Démocratique du Congo relance la reprise du processus de paix qui cumulera avec la signature des accords de Sun city, signés en Avril 2002, en Afrique du sud. Durant cette période, les représentants congolais, en étroite collaboration avec les bailleurs de fonds, multiplient les plans de stabilisation et de relance économique qui se caractérisent en trois niveaux essentiels tels que la participation très marquée des ins-

17 Dans un article intitulé « République Démocratique du Congo, l’heure du retour? », publié en 2003 dans African Mining, une revue du secteur de l'industrie minière d'Afrique du sud, on souligne ce retour en force des institutions multinationales en République Démocratique du Congo : Banque Mondiale, société financière internationale, Fond monétaire international, MIGA (Agence multilatérale de garantie des investissements). 
titutions financières internationales, l'ampleur des reformes et la rapidité de leur mise en application.

\section{III.2. L'IMPACT D'EXPANSION DE MININGS A KOLWEZI}

\section{III.2.1. L'impact social}

a) Des inconvénients

Les activités minières sont susceptibles d'entrainer un déplacement involontaire des populations de leur lien d'origine vers de nouveaux sites, créant de ce fait, des effets sociaux perturbateurs sur les familles et les résidents autochtones. Les projets miniers qui déplacent involontairement des populations donnent en général lieu à de sérieux problèmes économiques, sociaux et environnementaux.

Des systèmes de production sont démantelés, les biens des productions et les sources de revenu sont perdus, les populations sont déplacées dans des zones où leur capacité de production peut être inadaptée et où la concurrence pour les ressources sont plus rudes. Les structures communautaires et le tissu social peuvent être affaiblis : les groupes d'affinités peuvent être dispersés, l'identité culturelle et l'autorité traditionnelle peuvent être perturbée.

Le déplacement involontaire peut être une source de déficits à long terme en entrainant un appauvrissement des populations touchées et causer des dégâts dans les sites miniers. Il ya lieu de relever que l'afflux massif de populations diverses sur les sites, du à l'appétit d'un enrichissement facile et rapide, qui entraine également une dégradation de mœurs sur la plus part des sites miniers.

C'est ainsi que la prostitution, l'usage de stupéfiants, la délinquance, l'escroquerie, le banditisme, l'usage du chanvre et la criminalité, ont tendance à s'y développer. Ce danger est d'autant plus réel que souvent, il ya une nette insuffisance d'infrastructures sociales élémentaires aux plans santé, éducation et sécurité.

Une exposition humaine par inhalation des poussières ou ingestion des aliments contaminés par dispersion et transfert des métaux lourds principalement le cuivre par l'eau et le vent, est établie à proximité d'installations industrielles liées au cuivre. Les données récentes sur la santé et l'activité minière, montrent que les habitants de zones minières au Katanga et particulièrement à Kolwezi, sont exposés au danger d'intoxication par les métaux lourds.

En effet, les résultats ont relevés des niveaux élevés en cobalt, arsenic et plomb dans les urines des habitants qui résident à moins de quelques kilomètres des sites miniers ou industriels de Kolwezi. Et l'existence de relation dose-réponse renforce également l'hypothèse de causalité. En plus, l'on constaté que lorsque la pollution diminue suite à certaines interventions, il y a également réduction des effets sur la santé.

Un des éléments quelque peu troublants de ces observations est que les pics de pollution atmosphérique ont non seulement des effets respiratoire, mais aussi cardio-vasculaires, 
certes pendant les épisodes de pollution atmosphérique urbaine, il pourrait que plus de personnes meurent de maladies cardio-vasculaires que d'affection respiratoire. D'autres observations épidémiologiques indiquent que des paramètres cardio-vasculaires sont affectés par les polluants particulaires.

L'Etat congolais est tenu de prendre de mesures pratiques relatives à la l'encadrement Administratif et technique de la communauté locale, notamment le renforcement des mesures protectrices de leurs intérêts entant qu'entité composées d'éléments vulnérables, de la société, comme la création des comités locaux de développement pour assurer la gestion des affaires communautaires dans le domaine minier ${ }^{18}$.

$\mathrm{Au}$ lieu que l'exploitation minière soit une activité rémunératoire pour la communauté ou l'exploitation, apparait au contraire comme un facteur de paupérisation des zones rurales minières et l'enrichissement de ceux qui sont économiquement fort au détriment des faibles. L'exploitation minière par les privées, occasionne l'exode rural, les villageois abandonnent leur campagne, pour venir en ville aux fins d'exploiter les mines. Le secteur agricole est très négligé, d'où l'importation des produits vivriers.

\section{b) Des avantages}

Les potentialités offertes par l'exploitation minière sont des atouts favorables techniques et des collectivités de base en vue de faciliter leur engagement dans des activités productives durables. Améliorer l'accès aux services sociaux de la population et surtout répondant aux exigences, conformément aux lois nationales en vigueur; pour permettre à la base de s'adapter à la vie humaine pour le développement du pays en général et de la ville de Kolwezi en particulier.

L'exploitation minière constitue en effet, le principal moyen de substance dans la province et dans le district de Kolwezi. La plus part des travailleurs sont des hommes vivants dans les communautés avoisinant les zones carrières de Kolwezi, est une réponse qui resterait évidente à savoir, le facteur important est le contexte économique de la ville.

\section{III.2.2. L'impact économique}

L'investissement se révèle être le facteur par excellence de la croissance et efficacité économique, ce qui conduit forcement au développement, en ce qu'il consiste dans l'augmentation de grandeurs économiques. La croissance économique suppose elle-même des changements majeurs des structures et d'importantes modifications correspondantes dans les conditions institutionnelles et sociales du pays et dans le contexte mondial.

18 PERREAUX, F, Le multiplicateur d'investissement dans le pays sous développé un reçu du tiers monde, Paris, p67. 
a) Enjeux économique

Pays d'immenses ressources du sol et du sous-sol, objet des convoitises étrangères multiples, la République Démocratique Congo, pour assurer sa croissance économique ainsi que le bien-être de sa population doit aujourd'hui inscrire son avenir dans la logique de la conquête de l'économie mondiale.

Celle-ci, donne lieu chaque jour à d'intenses négociations sur toutes les questions de finance et de la bonne gouvernance économique. Les acteurs aux agents de la diplomatie économique étant désormais les entreprises multinationales, le gouvernement congolais doit non seulement intégrer les atouts économiques ou ses immenses ressources naturelles dans son ambition diplomatique mais plus se doter d'une politique des relations avec les entreprises multinationales ${ }^{19}$.

b) De la considération

La République Démocratique Congo étant un pays pauvre, dans un nombre riche et où la communauté internationale à travers les organisations mondiales pour le développement, demande à tous les Etats de prioriser le développement socio- économique durable, Elle doit chercher à attirer les investisseurs étrangers tant privés que publics dans l'espoir ainsi d'enrichir le monde tout en s'enrichissant d'elle-même.

Cela suppose une politique volontariste et d'une appropriation nationale de la mondialisation et de la globalisation; soit une addition aux ressources d'une vision et d'une intelligence dans le domaine économique. Les acteurs économiques devront œuvrer de concert avec les représentants de la diplomatie congolaise dans le monde pour permettre à l'économie nationale d'augmenter rapidement et répondre à l'exigence de la population.

La voie pour y parvenir, est la coopération internationale, appliquée au contexte particulier de la République Démocratique Congo, pays en guerre économique; les pillages de substances minérales, la chute de la Gécamines et autres infrastructures, ont fait qu'elle tombe dans une crise économique incroyable. Mais chose étonnante qu'en 2002 une aire d'innovation des entreprises minières dans la ville de Kolwezi qui ont mise en œuvre leurs capitaux et qui ont favorisé à une inflation de l'économie.

\section{III.2.3. L'impact environnemental}

Dans le domaine minier, les normes environnementales sont régies par le code et le règlement minier. L'article premier du nouveau code minier en son alinéa 19, dispose que : «l'analyse scientifique préalable des impacts potentiels prévisibles d'une activité donnée sur l'environnement dans les limites de meilleurs technologies disponibles à un cout économique viable». son alinéa 40, définit le plan d'atténuation et de réhabilitation comme étant «plan requis pour les opérations en vertu d'un droit minier ou de carrière, de re-

19 http:// mémoire online. Com/, « la problématique de l'exploitation minière artisanale », 2012, p5. 
cherche ou d'une autorisation d'exploitation de carrières temporaires, consistant dans l'engagement du titulaire de réaliser certaines mesures d'atténuation des impacts de son activité sur l'environnement, ainsi que des mesures de réhabilitation, constitue une sureté financière pour garantir le coût d'atténuation de l'environnement».

De cette disposition, il découle que le titulaire n'est pas seulement tenu de respecter les engagements pris en matière de protection de l'environnement, mais également de fournir une sureté financière représentant la garantie du coût de processus d'atténuation et de réhabilitation.

a) Pollution

L'acheminement de matériels sur les sites des activités, peut affecter l'environnement lorsque les sites sont localisés dans la zone écologiquement sensible. Cette perturbation est moindre par rapport à la perturbation qui s'applique à l'ensemble de l'activité minière.

Les activités minières sont susceptibles d'avoir l'ampleur d'influence plus grande sur l'environnement, c'est pourquoi elles doivent être menées en tenant compte de tous les aspects liés à l'environnement. Voila pourquoi le principal impact à l'environnement causé par les opérations de prospection, est la perturbation du relief dû à la construction des routes, des plates- formes de sondage, ainsi qu'à l'utilisation des matériels lourds.

L'exploitation minière est susceptible de provoquer une contamination de l'air par les poussières provenant du déplacement des roches et par les gaz suite à l'utilisation des explosifs, perte de la faune et végétaux qui entrainent un important déséquilibre de l'environnement, le déboisement excessif, d'où la genèse de la sécheresse, d'érosion, etc. L'exploitation minière est également l'objet de la contamination radioactive de minerais uranifères qui contribue à la destruction de l'humanité.

Le constat fait est que les services géologiques nationaux, ne disposent pas des moyens et même de spécialistes, capables d'appréhender tous les aspects liés à l'étude et à la protection de l'environnement. Dans la politique de relance du secteur minier, il s'agira de concilier une production génératrice du revenus et d'emplois pour le désire légitime de maintenir un environnement sain dans la ville minière de Kolwezi.

Parmi les impacts prévisibles associés à l'exploitation d'une mine, qu'elle soit à ciel ouvert ou à carrières, on retiendra les perturbations et les déséquilibres qui sont susceptibles d'affecter non seulement l'écosystème mais aussi la nature et l'habitat de la faune, la dégradation du sol et du couvert végétal et la perte de vue panoramique ${ }^{20}$.

20 KATEMO, M.B., Evaluation de la contamination de la chaine trophique par les métaux lourds dans le bassin de la Lufira Supérieure(Katanga) R.D.Congo, mémoire de D.E.A. en droit, géographie, histoire,....UNILU, 2009, pp31-32. 


\section{b) L'intoxication}

Une mine en activité ou abandonnée, peut être une source d'accident lié à la circulation d'engins lourds ou à l'émanation de gaz toxique qui est particulièrement le cas pour les mines de charbon. Ces gaz ou mélanges de gaz sortant d'un puis peuvent présenter également des risques d'asphyxie, de toxicité, d'inflammabilité ou d'explosion. L'application des mesures de protection collective ou individuelle et le respect de normes de sécurité et d'hygiène, sont en général les moyens les plus utilisés et essentiels dans les mines pour circonscrire les risques d'accidents professionnels.

Les exploitants miniers entreposent les produits miniers dans des emplacements non préposés et inappropriés, notamment dans les résidences familiales, ceux qui ont des dépôts dans les quartiers industriels ne respectent guère les techniques et équipement d'entre posages, de forme, hauteur, le volume de monticules des produits miniers exigés par les dispositions de l'article 4 du règlement minier à l'annexe IV.

\section{c) Effets}

S'agissant de la pollution atmosphérique, il ya lieu de rappeler que les normes de rejets dans l'atmosphère imposées aux industries et les changements de sources énergétiques ont permis de réduire considérablement la pollution urbaine apparait désormais également sans forme d'une pollution photo-oxydante ${ }^{21}$.

\section{DES ENQUETES DE LA PROTECTION DE LA MAIN D'CEUVRE PAR LES MININGS A KOLWEZI}

En 2010 les embauchés étaient au nombre de 6.606 hommes et 153 femmes, dont 39 hommes et 10 femmes licenciés; 16 hommes et 5 femmes ont démissionné; 16 hommes et une femme sont arrivés à l'expiration normale de leur contrat; 15 hommes et 16 femmes salariés décédés; 206 hommes et 25 femmes chômeurs (les non universitaires : 148 hommes et 14 femmes et les universitaires : 58 hommes et 11 femmes).

En 2011 les embauchés étaient 3625 hommes et 181 femmes, dont 119 hommes et 4 femmes, 65 hommes et 2 femmes ont démissionné; 20 hommes et zéro femme décédés; 527 hommes et 7 femmes sont arrivés à l'expiration normale de leur contrat; 904 hommes et 53 femmes chômeurs (les non universitaires : 43 hommes et 20 femmes et les universitaires : 153 hommes et 33 femmes).

En 2012 les embauchés étaient 1163 sans déterminer, dont 69 licenciés, 27 ont démissionné; 4 décédés; 8711 hommes et 1243 femmes chômeurs (Les non universitaires : 7656 hommes et 985 femmes et Les universitaires : 1055 hommes et 259 femmes) ${ }^{(46)}$.

21 W.W.W.egmforrest.com/MUNENGE Chef du personnel. 
En 2013 les embauchés (pas de détermination du nombre), dont 89 licenciés en générale; 5 décédés; 3701 hommes et 221 femmes chômeurs (les non universitaires : 2922 hommes et 112 femmes et les universitaires : 779 hommes et 109 femmes).

\section{IV.1. ETAT DE LA QUESTION AU SEIN DE L'ENTREPRISE MALTA FOREST}

\section{IV.1. 1. Présentation}

a) Aperçu historique

Créée par Monsieur Malta Forest, le 22 février 1922 dans la province du Katanga au Congo-belge. L'entreprise générale Malta Forest (E.G.M.F. en sigle) est la plus ancienne et la plus expérimentée de la région dans son domaine d'activité. Société de transport à l'origine, elle s'oriente rapidement vers le secteur minier et exploite, dès 1933, l'Or dans la région de Kolwezi, de 1937 à 1939, elle met en valeur les mines de cuivre de Musonoie et exploite parallèlement, en 1939, la mine de manganèse de Kasekelesa dans le sud Katanga.

De 1951 à 1954, l'entreprise participe au démarrage de la mine de manganèse de Kisenge tant en exploitation minière qu'en travaux de génie civil. A cette époque, le comité spécial du Katanga confie à l'entreprise, la construction de tout le réseau d'égouts et de voirie de la ville de Kolwezi, ainsi que son aérodrome. C'est à ce moment qu'elle se convertit en entreprise de génie civil. Elle va alors jouer un rôle important dans l'édification des infrastructures de la région cuprifère de l'ouest du Katanga et continuera inlassablement ses activités au travers de toutes les crises politiques et économiques que connaitra le pays après son indépendance.

Dès 1990 et pour compte de la Gécamines, l'entreprise effectue d'importants travaux de découverture minière à Kolwezi; cinq millions de mètre $\left(\mathrm{m}^{3}\right)$ par an avec un objectif de douze millions de $\mathrm{m}^{3}$, d'un important programme de réhabilitation desvoiries.

\section{b) Fonctionnement}

L'entreprise Malta Forest effectue de grands travaux de terrassement exécutés généralement en sous-traitance de l'industrie minière, voiries urbaines et assainissement, les travaux de réhabilitation de route en terre. Elle est également focalisée à la préparation d'enrobée pour l'asphaltage des routes, construction de routes asphaltées, les travaux de bâtiment et d'ouvrage d'art tels ceux de Kamina, ainsi que le canal d'alimentation de centrale hydroélectrique de Kilubi, l'adduction d'eau.

L'activité minière comprenant le minage, l'exhaure, la découverte, le changement et le transport des minerais et du stérile ainsi que la gestion des remblais. La production de ballast de pierrailles calibrées incluant les travaux de carrière (minage, découverture, chargement) le concassage et le criblage et aussi le lavage de gravier (carrière à Kipushi et Katon- 
to) ses activités tendent à la préfabrication de briques et de blocs en ciment, de brises et d'éléments de voirie en béton; de trading à Kalemie ${ }^{22}$.

c) Protection de la main-d'œuvre

Selon nos recherches effectuées, l'entreprise générale Malta Forest est constituée en son sein, de 21 cadres, 149 maitrises, 588 ouvriers et 20 expatriés. L'ensemble est 810 comme main d'œuvre. Parmi ces expatriés, les uns œuvrent au siège social se trouvant à Lubumbashi et les autres sont de superviseurs dans le site d'exploitation. Nous référant à l'arrêté ministériel $\mathrm{N}^{\circ} 121 \mathrm{COB} / \mathrm{PTS} / 112 / 2005$ du 26/ octobre 2005 fixant, le pourcentage autorisé des employés étrangers au sein d'une firme, à $5 \%$; nous nous rendons compte que, le pourcentage des étrangers dans cette entreprise, est d'environ $2.5 \%$ en deçà même du pourcentage légal.

\section{IV.2. LA SOCIETE KATANGA METALS (S.P.R.L)}

\section{IV.2.1. Présentation}

La société Katanga Metals a été créée par l'initiative de Madame Mireille SENGA Pillai, de nationalité congolaise, née à Lubumbashi; le 16 juillet 1977 et par Monsieur Aman Datwani, de nationalité indienne, ne à Hong Kong, domicilié au siège social de la société à Lubumbashi où il est constitué. Le siège social de la société Katanga Metals est établi sur l'avenue Kapenda ${ }^{\circ} 191$ dans la commune et ville de Lubumbashi. Ce siège pourra être transféré à tout autre endroit de la République Démocratique Congo, que sur décision de l'assemblée générale. La société a pour objet la production, l'exploitation, la transformation et la commercialisation des substances minérales ainsi que l'exécution des travaux de génie civil.

La gérance de la société peut établir des sièges d'exploitation succursales, bureaux,...à n'importe quel endroit du Pays ou à l'étranger. Elle pourra s'intéresser par voie d'apport, de fusion, de souscription, d'intervention financière ou de toutes autres manières à toutes les entreprises ou sociétés ayant en tout ou en partie un objet similaire ou connexe ou sein. Elle pourra également participer à toutes opérations qui directement ou indirectement sont en rapport avec objet social et qui sont de nature à favoriser l'accroissement de son patrimoine. La société est constituée pour une durée indéterminée prenant cours à la date de son immatriculation au nouveau registre de commerce en 2005.

\section{IV.2.2. Organisation et fonctionnement}

Tout au long de l'année 2013, la société Katanga Metals a mis à la disposition de son personnel au moyen de transport constitué deux bus de capacité moyenne de trente places cha- 
cun, de deux mini bus et d'un jeep land cruiser, il faudra ajouter les indemnités de transport qui sont payés à tout son personnel pour permettre à ses agents d'attendre les différents de ramassage ou de stationnement des bus.

Dans ses efforts, l'entreprise a procédé par l'embellissement de bâtiments administratifs publics, une aide financière pour l'asphaltage de voies routières, de sécurité du travail et travailleur; l'entreprise Katanga Metals a fourni beaucoup d'efforts non seulement pour améliorer et assainir les conditions de travail mais également réduire sensiblement les risques des accidents et le nombre excessif de chômeurs dans la ville minière de Kolwezi.

A cet effet, le personnel a été équipé plusieurs fois d'outils nécessaires pour le travail, un coin de secours pour les premiers soins existe, des réunions du comité chargé de l'hygiène et sécurité du travail sont organisées et l'entreprise a enregistré pour l'année 2013, trois cas d'accident légers. Au cours de cette année, la société a souscrit un abonnement de tout le personnel aux centres de santé clinique Nazaire et Uzima à Luilu qui sont estimés à environ 30.000.000FC pour toute l'année.

L'entreprise a initié comme d'habitude plusieurs actions au bénéfice de Kolwezi et de ses environs; allant de l'assistance ponctuelle aux coté de la Mairie de Kolwezi à l'achèvement de la construction d'une habitation pour le chef Samukinda. L'entreprise constate dans le fonctionnement de ses activités une production consistante et rémunératrice et surtout un entretien au cours de l'année 2013, les relations franches et harmonieuses qui ont caractérisé les rapports entre l'employeur et le banc syndical.

\section{IV.2.3. Protection de la main d'œuvre}

Voici l'effectif général du personnel : A la date de clôture de l'exercice soit le 31/12/2013, la société a enregistré 97 chefs dont, 83 nationaux et 14 étrangers. Par catégorie professionnelle, on a six comme les cadres de direction, un cadre de collaboration, 16 maitrises, 9 hautement qualifiés, 16 qualifiés et enfin 49 agents ${ }^{23}$.

\section{RECOMMANDATIONS}

La ville minière de Kolwezi enviée par la puissance du monde entier, actuellement elle traverse une situation très chagrinée dans la mesure où elle ne sait pas se maintenir. Par ailleurs, sachant que le monde actuel est celui de l'investissement de substances tant minérales, pétrolières qu'agricoles, faute de quoi, ça pourrait entrainer un sous développement très avancé.

En effet, pour éviter qu'elle soit en cascade, le premier palliatif est celui de permettre, à tout particulier qui possède un moyen consistant d'obtenir un permis d'exploitation et ce dans le but de promouvoir la politique d'emploi, l'investissent et la protection de la main-

23 Direction des ressources humaines. 
d'œuvre. Pour y arriver, il est important de veiller surtout au respect de la loi et au renforcement du contrôle efficace dans les sites tant minier, agricole que pétrolier.

Par ailleurs, les agents de services d'immigration, doivent veiller au contrôle du taux plus élevés des expatriés dans les sociétés minières qui mettent à genoux l'arrêt départemental 86/001 du 31 mars 1986 déterminant la liste des emplois interdits aux étrangers.

Le gouvernement congolais, devra exactement mettre en œuvre des mesures prompte de façon que celui qui enfreindrait les règles juridiques en vigueur. Sanctionné sévèrement conformément à la loi congolaise. L'Etat doit normalement ouvrir le secteur minier à tous les investisseurs sous les mêmes règles de jeux, éliminer toute sorte de discrimination dans l'octroi des droits miniers entre les entreprises publiques et sociétés privées et appliquer à cet état de chose des critères : premier venu, premier suivi; garantir le droit de poser de la recherche à l'exploitation minière sous conditions claires et objectives, libéraliser la mutation des droits miniers et carrières, instaurer des sûretés minières et des conditions permettant l'accès facile de financement d'un pays minier.

Faut-il protéger la main d'œuvre locale, alors que les compétences vous pour le développement des entreprises ne trouvent qu'à l'étranger. En son temps, la Générale des carrières et des mines (G.C.M. en sigle), organisait des écoles de formation de toutes les compétences dont elle avait besoin. Ces écoles aujourd'hui à Kolwezi, sont par terre à cause de sa chute. Il est alors impérieux que l'Etat lui-même prenne la relève pour organiser des écoles en vue de former les travailleurs selon les attentes des entreprises. De cette manière, on pourrait consolider la politique d'emploi et la protection de la main d'œuvre locale par rapport à la concurrence de la main d'œuvre étrangère.

\section{CONCLUSION GENERALE}

Il convient de dire que tout au long de notre recherche relative à notre sujet : " l'exploitation minière et la protection de la main d'œuvre locale à Kolwezi », nous avons été préoccupé par un problème majeur qui est celui de savoir pourquoi dans la ville minière de Kolwezi il ya un développement plus remarquable des sociétés minières et ses inconvénients dans la circonscription de la ville de Kolwezi.

Pour ce faire, nous avons défini de concepts clés qui ont constitué notre travail, nous avons aussi démontré les causes d'expansion des sociétés minières à Kolwezi, toujours dans cet état de chose nous avons parlé de l'histoire du code minier jusqu'au nouveau code minier de 2002, son importance. Nous nous sommes forcés de faire une enquête dans quelques sociétés minières énumérées dans ce travail pour savoir et comprendre si réellement ces dernières, respecte la loi en vigueur.

Nous avons constaté que dans certaines sociétés, le quota de la main d'œuvre étrangère tel que réglementé, est respecté. Mais la où la main d'œuvre locale est utilisée selon la loi, l'on a constaté que les travailleurs font presque du bénévole au profit des multinationales. Tous ceux qui sont recrutés, peu sont ceux qui sont considérés selon leur niveau d'études et payés en conséquence. 
Ces considérations nous ont poussées à interpeler l'Etat Congolais, à travers l'implication des organes locaux, à assurer un suivi nécessaire aux fins de répondre aux exigences de la protection de la main d'œuvre nationale. Pour ne pas asphyxier les multinationaux, il est nécessaire que l'Etat veille à la formation des nationaux appelés à œuvrer dans les minings, selon les attentes des exploitants miniers étrangers ayant investi en République Démocratique du Congo.

\section{BIBLIOGRAPHIE}

Textes de lois

1. la constitution de R.D.C. du 18/06/2006.

2. O-L. du 06/06/1974, portant protection de la main d'œuvre nationale

3. La convention du 25 juin 1958 concernant la discrimination en matière d'emploi et de profession.

4. Arrêt départemental du 30 octobre 1975, 27/75 fixant les modalités de la réclamation annuelle de la situation de la main d'œuvre J.O.Z. N4-15/02/1976.

5. Arrêt départemental $067 / 81$ du 05 novembre 1981, réglementant le transfert à la l'étranger de la main d'œuvre nationale J.O.Z. N01/01/1982.

6. Lettre 12/D.T.P.S/ CAB/0730/83 protection de la main d'œuvre nationale du 13 août 1983.

7. Article 116 du Code minier Malien.

8. décret $\mathrm{N}^{\circ} 038 / 2003$ du 26/03/2003 portant règlement minier, J.O.R.D.C.

9. Article 3 de la loi $\mathrm{N}^{\circ} 004 / 2002$ du 21/02/2002 portant code des investissements de la R.D.C.

10. ordonnance -loi $\mathrm{N}^{\circ} 63$ du 10 février 1969 portant code foncier et immobilier.

11. Code larcier, droit commercial et économique, T3 Vol2 Afrique, éd Bruxelles 2003.

12. Exposé de motif de la constitution du 18/02/2006.

13. La loi $\mathrm{N}^{\circ} 007 / 2002$ du 11/07/2002 portant code minier, in J.O.N spécial, 15/07/2002.

Ouvrages, revues et autres documents

1. Paul Palster Man et Alii, Guide de la législation sociale, éd, syndicaliste, 2003.

2. MUKADI Bony, Droit du travail CR. DJ, 2008.

3. Guillien et Vincent, J, Lexique des termes juridiques, éd Dalloz, paris.

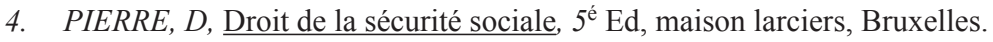

5. KATAMBWE Mutombo, Législation minière congolaise, business service, Kin 1999.

6. CAZIN, $M$, les mines presses universitaires de France, collection « qui sais-je? 》, paris, 1951.

7. MUKENDI WA FWANA, E, Droit minier congolais, jur.congo, Kin.

8. BONNIE Camp Bell, En jeux des nouvelles règlementations minières en Afrique 2004. 
9. MATON, J, Solignacle Comte, H, Les espoirs reçus du « Brésil Africain » document de travail

10. Nemmar; a, Hoet, p.h.m., Nemmery. B., "les épisodes de pollution atmosphérique et leurs effets sur la santé." In Mail, respectiv, Belgique, 2003.

11. Perreaux, $F$, Le multiplicateur d'investissement dans le pays sous développé un reçu du tiers

12. KATEMO, M.B., Evaluation de la contamination de la chaine trophique par les métaux lourds dans le bassin de la Lufira Supérieure(Katanga) R.D.Congo, mémoire de D.E.A. en droit, géographie, histoire, .... UNILU, 2009.

13. W.W.W.egmforrest.com/MUNENGE CHEF DU PERSONNEL

14. http:// mémoire online. Com/, " la problématique de l'exploitation minière artisanale ", 2012.

15. Dictionnaire petit Larousse, 114, paris.

16. N¹78 centre de développement de l’OCDE, 1999. 